\title{
Prediction of the Effect of Defect Parameters on the Thermal Contrast Evolution during Flash Thermography by Finite Element Method
}

\author{
Maodan Yuan*, Hu Wu*, Ziqiao Tang*, Hak-Joon Kim*, Sung-Jin Song* ${ }^{\dagger}$ and Jianhai Zhang*
}

\begin{abstract}
A 3D model based on the finite element method (FEM) was built to simulate the infrared thermography (IRT) inspection process. Thermal contrast is an important parameter in IRT and was proven to be a function of defect parameters. Parametric studies were conducted on internal defects with different depths, thicknesses, and orientations. Thermal contrast evolution profiles with respect to the time of the defect and host material were obtained through numerical simulation. The thermal contrast decreased with defect depth and slightly increased with defect thickness. Different orientations of thin defects were detected with IRT, but doing so for thick defects was difficult. These thermal contrast variations with the defect depth, thickness, and orientation can help in optimizing the experimental process and interpretation of data from IRT.
\end{abstract}

Keywords: Infrared Thermography, Thermal Contrast, Finite Element Method, Defect Orientation

\section{Introduction}

Recent years, infrared thermography (IRT), which is also known as thermal inspection or infrared imaging, has attracted a lot of attentions on the nondestructive test (NDT) fields [1,2]. As the most conventional NDT methods, like ultrasonic, magnetic field and eddy current methods have two significant drawbacks: physical contact to the material under test is required and they generate images slowly by scanning, and then they are mainly suitable for the detection of defects at depths from 5 to $100 \mathrm{~cm}$. However, the IRT gives immediate information rapidly with relatively low cost and it is becoming more and more widely used along with other NDT methods [2-4].

In the case of IRT, the remarkable strengths are as following: fast inspection rate, no couplant needed and no harmful radiation involved [1]. Although IRT is not good at the effects of thermal losses, which induce spurious contrasts affecting the reliability of the interpretation, IRT still have the bright future and is generally used for the non-contact inspection of structures. The results are recorded by thermal and infrared sensors in order to visualize thermal surface contrast after a thermal excitation. IRT measures the radiated electromagnetic energy in the infrared zone of the electromagnetic spectrum and produces 2D-images that represent heat wave contour. According to the electromagnetic spectrum, there are three wave infrared bands: the short $(0.9-1.7 \mu \mathrm{m})$, mid $(3-5 \mu \mathrm{m})$ and long $(8-14 \mu \mathrm{m})$ region. The camera wavelength should be chosen carefully for the surface measurement, because the mid wave camera can receives emission from both the coating surface and the internal matrix. Therefore, the long wave infrared camera is preferential for surface measurement [5]. Furthermore, IRT with a selective heating of the surface under investigation, permits to detect and to characterize the non-homogeneities in buildings structures in the near surface region up to a depth of about $10 \mathrm{~cm} \mathrm{[6-8].}$

The main principle of IRT is that the thermal energy propagates by diffusion under

[Received: December 5, 2013, Revised: January 2, 2014, Accepted: January 7, 2014] *Sungkyunkwan University, School of Mechanical Engineering, Suwon, 440-746, Korea †Corresponding Author: sjsong@skku.edu

(c) 2014, Korean Society for Nondestructive Testing 
the surface while a thermal infrared camera monitors and records the temperature variation over the viewed surface. Optical excitation techniques [1,7-9], including pulsed and modulated thermography, are the most common excitation techniques used in thermography. Other excitation methods include electromagnetic induction [5], mechanical stimulation like sonic or ultrasonic $[3,4]$, and chemical excitation like exothermic reaction of binary adhesive. In case of homogenous material and uniform surface heating, temperature is uniformly distributed on the material under test. The presence of a defect at a certain depth interferes with the heat flow causing local surface temperature variations or any other change in the thermal properties of the material. The changes in heat flow cause localized energy differences on the surface of the test object, which can be measured using an infrared detector. The thermal signatures are then used to evaluate various flaws, including leaks, cracks, debonding, corrosion, poor electrical wiring and contacts, and delaminations. Thermographic NDT method typically involves the evaluation of thermal signatures of material under test using imaging techniques [2]. The most highlight feature of the thermal image technique in contrast to other NDE techniques is that it facilities evaluating larger parts of the structure in a single. Many researchers developed new approaches for analyzing transient temperature data $[9,10]$. Titman [1] evaluated some applications of the thermal imaging technique in civil structures such as ascertaining the degree of thermal insulation, detecting entrapped moisture and locating buried pipelines, using solar heat as the natural sources. Cheng et al. [8] used the fusion of information gathered from active IRT and elastic waves for the inspection of building exteriors. Sakagami et al. introduced a quantitative technique for delamination detection by the phase delay measurement under periodical heating [9]. Kim et al. developed a robust analytic approach to detect surface cracks using the contour integral of holomorphic function under steady-state thermal conditions [10]. Cho utilized lock-in infrared thermography to optimize phase comparative images in detecting defects in welded part of ship and offshore structures [11].

Numerical simulation is expected to predict the experimental results with certain condition that the experiments are difficult to implement and cost too much. Modeling of IRT can help us to obtain the physical insight of the thermal phenomena occurring during and after thermal excitation of structures and fully understand all the aspects of their thermal behavior $[7,12,13]$. In order to investigate the efficiency and the feasibility of IRT, it is necessary to take the internal defect characteristics into consideration. The depth, thickness, and orientation are the common parameters of the defect. These three parameters may influence the heat transfer in the specimen during the IRT process, thus have been studied in the present work. Based on this study, the results are potential to optimize the system performance to enhance IRT effectiveness in internal structures detectability.

In this paper, a FEM-based model has been applied to simulate the thermal phenomena in IRT. A brief model description for IRT is shown in part 2. The thermal contrast is considered to be the discriminating parameter and the parametric studies of defects with different depths, thickness and orientations are implemented in part 3. Finally, the conclusion is made in part 4.

\section{Model Descriptions}

The numerical simulation of the IRT is implemented through solving the heat transfer equation with the commercial software COMSOL multiphysics. 


\subsection{Governing Equation and Boundary Conditions}

The underlining physics in heat transfer is governed by the partial differential equation:

$$
\rho C_{p} \frac{\partial T}{\partial t}-\nabla \cdot(k \nabla T)=0
$$

where $\rho$ is the density $\left(\mathrm{kg} / \mathrm{m}^{3}\right) ; C_{p}$ refers the material heat capacity at constant pressure $(\mathrm{J} /(\mathrm{kg} \cdot \mathrm{K})) ; \quad T$ is absolute temperature $(\mathrm{K}) ; k$ indicates the material thermal conductivity $(\mathrm{W} /(\mathrm{m} \cdot \mathrm{K})) ; t$ refers to time.

The initial condition is represented by the ambient temperature, shown as:

$$
T(x, y, z, t=0)=T_{a m b}=293.15[K]
$$

where $T_{a m b}$ refers to the ambient temperature.

The boundary conditions are heat transfer by convection and radiation from the corresponding surfaces, shown as following:

$$
\mathbf{n}(k \nabla T)=q_{0}+h_{\text {conv }}\left(T_{a m b}-T\right)+\sigma \varepsilon\left(T_{\text {amb }}^{4}-T^{4}\right),
$$

where $q_{0}$ refers to the heat source (W); $h_{\text {conv }}$ is the constant convective heat transfer coefficient $\left(\mathrm{W} /\left(\mathrm{m}^{2} \cdot \mathrm{K}\right)\right) ; \sigma$ is the Stefan-Boltzmann constant $\left(\mathrm{W} /\left(\mathrm{m}^{2} \cdot \mathrm{K}^{4}\right)\right) ; \varepsilon$ is the emissivity that is the ratio of radiant emittance of an object to that of a blackbody at the same temperature. The second and third terms are accounting for the convection and radiation heat fluxes respectively.

\subsection{Model Setup}

The 3D model geometry with adiabatic condition at lateral surfaces is consisted of a rectangular plate with defects and two halogen lamps, shown in Fig. 1. The distance of the internal defect with a thickness $t$ from the plate surface is denoted by $d$, and the defect can rotate with an angle $\theta$ to investigate the orientation effect. The center and the edge point are used to extract the surface temperature of the defect $T_{d}$ and host material $T_{s}$, respectively.

The model simulates the heat source by two halogen lamps, which is shown in the Fig. 1 (b), with $500 \mathrm{~W}$ each of them. The material properties from [7] are applied and summarized in table 1. In order to simplify the analysis, the

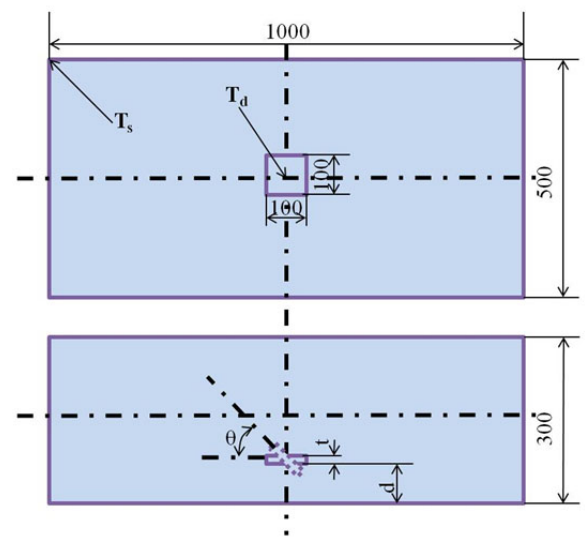

(a)

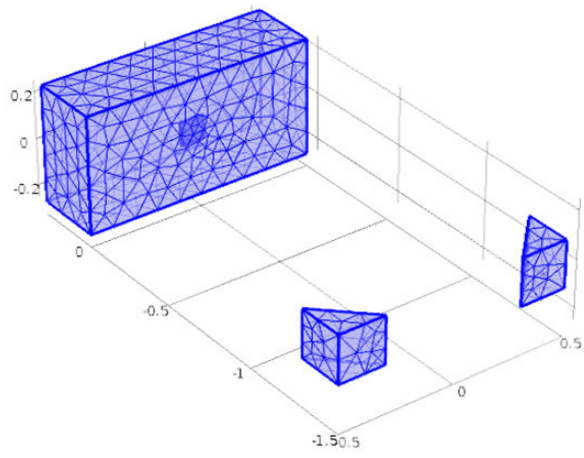

(b)

Fig. 1 Geometrical models: (a) 2D dimension of specimen and (b) meshed 3D model of specimen with defect and two halogen lamps

Table 1 Material property for simulation

\begin{tabular}{|c|c|c|}
\hline & Concrete & Halogen Lamps \\
\hline $\begin{array}{c}\text { Thermal Conductivity, } \\
\mathrm{k}[\mathrm{W} /(\mathrm{m} \cdot \mathrm{K})]\end{array}$ & 1.8 & 400 \\
\hline $\begin{array}{c}\text { Heat Capacity, } \\
\mathrm{C}_{\mathrm{p}}[\mathrm{J} /(\mathrm{kg} \cdot \mathrm{K})]\end{array}$ & 1000 & 10 \\
\hline Density, $\rho\left[\mathrm{kg} / \mathrm{m}^{3}\right]$ & 2300 & 8700 \\
\hline Surface Emissivity, $\varepsilon$ & 0.92 & 0.99 \\
\hline
\end{tabular}


space between the concrete and the halogen lamps is set as empty space.

\section{Parametric Studies of IRT}

A series of parametric studies have been implemented to obtain a full understanding of the IRT. At first, the thermal contrast will be calculated to obtain the maximum thermal contrast. Then, different depth $d$, thickness $t$ and orientation $\theta$ of the defects will be investigated to see the thermal contrast change.

\subsection{Thermal Contrast Calculation}

Here a total irradiation time of $15000 \mathrm{~s}$ has been investigated. The surface temperature from the solution is shown in Fig. 2. Also, the power curve of the halogen heat source of $500 \mathrm{~W}$ has been obtained in Fig. 3 .

Thermal contrast is one of the most important parameters in the infrared thermography inspection and various definitions exist [14]. The standard thermal contrast used in this work is expressed as:

$$
C_{T}(t)=\frac{T_{d}(t)-T_{s}(t)}{T_{s}(t)-T_{s}(0)}
$$

where $C_{T}$ is the thermal contrast; $T_{d}$ is the surface temperature of the defect; $T_{s}$ is the surface temperature of the host material; $T_{s}(0)$ is the ambient temperature.

In the FEM simulation, the surface temperatures on defect and host material can be extracted, shown in Fig. 1(a). The surface temperatures and the corresponding thermal contrast revolution with irradiation time are obtained as Fig. 4.

From the simulation result, at short irradiation time condition (smaller than $1000 \mathrm{~s}$ ), some noises exist, which should be avoided in IRT inspection, and the thermal contrast will increase with

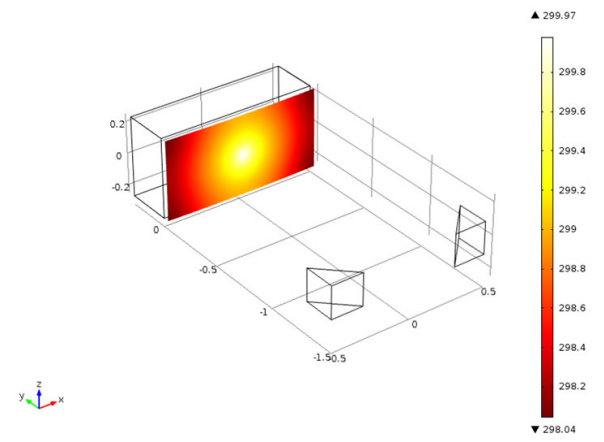

Fig. 2 The simulation result for the surface temperature

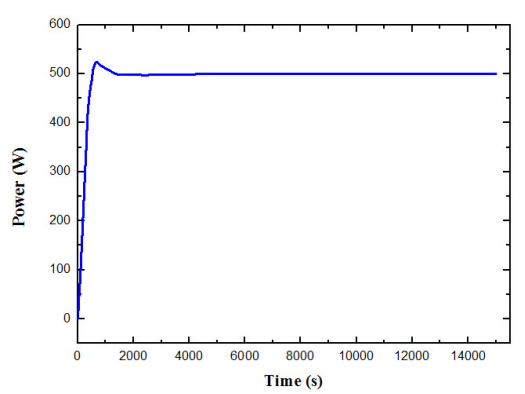

Fig. 3 The power curve of a halogen heat source of $500 \mathrm{~W}$

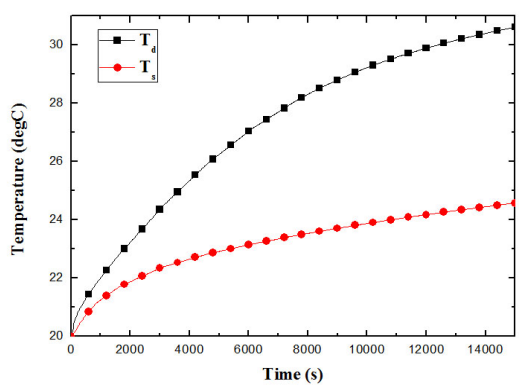

(a)

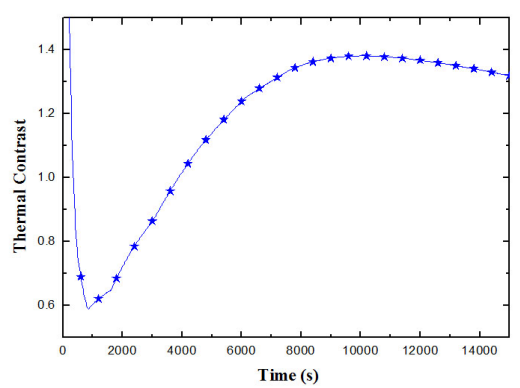

(b)

Fig. 4 The evolution with irradiation time: (a) for surface temperatures of defect and host material, and (b) for the corresponding thermal contrast 
increasing irradiation time. At some irradiation time, the thermal contrast reaches a maximum value, which is the most effective point for IRT inspection. Under this case, the irradiation time $t_{\max }$ corresponding to the maximum thermal contrast $C_{T, \max }$ is $10000 \mathrm{~s}$.

\subsection{Defects with Different Depths}

The same structure defect with various distances from the surface can also have a big influence on the IRT results. The thermal contrast has been calculated under the same procedure mentioned in section 3.1 and the results are shown in Fig. 5.

The simulation results show that with the increasing of the defect depth, the thermal contrast decreases and the irradiation time $t_{\max }$ for the maximum contrast increases $C_{T, \max }$ at

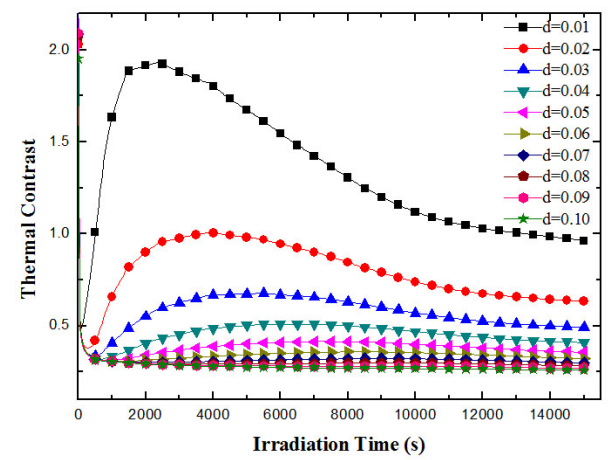

Fig. 5 The thermal contrast evolution with time for different defect depths

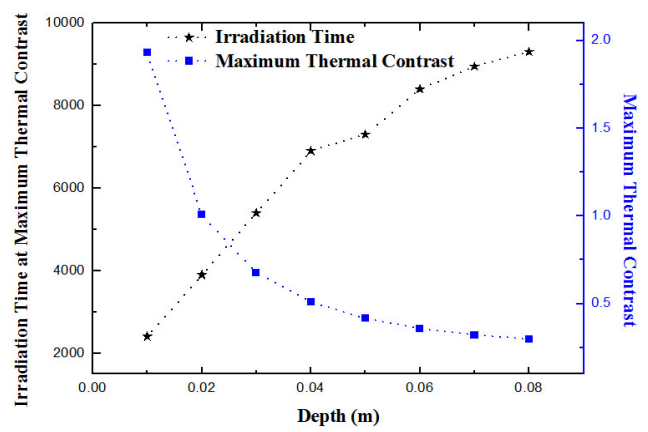

Fig. 6 The maximum thermal contrast and its corresponding irradiation time variations with defect depths the same time. The maximum thermal contrasts and their corresponding irradiation time have been expressed as Fig. 6 .

From the results, the maximum thermal contrast shows an inverse relationship with the defect depth while the corresponding irradiation time is almost linearly related with defect depth.

\subsection{Defects with Different Thicknesses}

The defects with different thicknesses at the given depth have also been taken into consideration. The thermal contrast varying with time has been shown as Fig. 7.

From Fig. 7, the thermal contrast shows little difference of the evolution in time domain. The maximum thermal contrast and its corresponding irradiation time have been analyzed and the results are shown in Fig. 8.

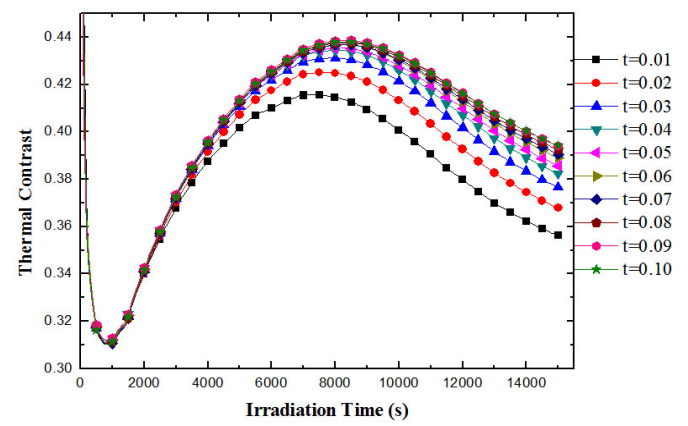

Fig. 7 The thermal contrast evolution with time for different defects thicknesses

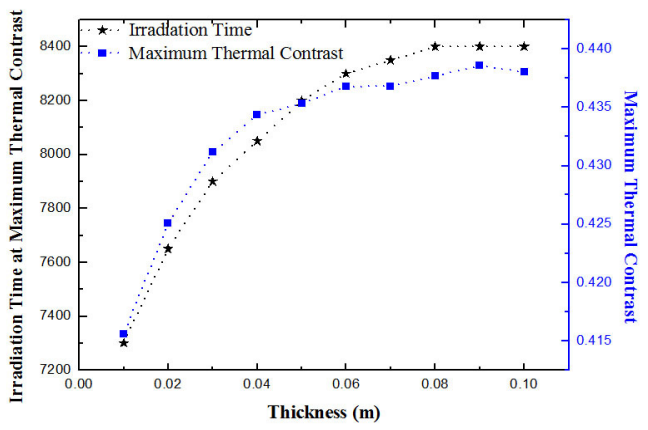

Fig. 8 The maximum thermal contrast and its corresponding irradiation time variations with different defect thicknesses 
From the results, both the maximum thermal contrast and its corresponding time increase with defect thickness increasing and becomes saturated around $0.08 \mathrm{~m}$. However, the variation is very small and it is hard to be distinguished during the IRT experiment. Therefore, the result proves that the IRT is not good at measuring the defect thickness.

\subsection{Defects with Different Orientations}

When the center of the defect is fixed, rotating the defect inside the specimen can give the influence of thermal contrast of different defect orientations. The relation between thermal contrast and the orientation $\theta$ is shown in Fig. 9.

From Fig. 10, the thermal contrast increases with the increased defects orientation, while the corresponding irradiation time decreases. In order to investigate this phenomenon, several different thickness defects are taken into consideration and the maximum thermal contrasts are compared in Fig. 11 and Fig. 12.

From Fig. 11 and Fig. 12, the influence of defect orientation is highly related with the defect thickness as well. For the ultra thin defect $(\mathrm{t}=0.01 \mathrm{~m})$, the maximum thermal contrast increases with the increased defect orientation, while the corresponding time decrease. For the thick defect $(\mathrm{t}=0.10 \mathrm{~m})$, the variation in thermal contrast is very small and irregular. This phenomenon is assumed to be related with the distance from the irradiated surface to the nearest part of the defect.

\section{Conclusion Remarks}

In this work, the computation with the $3 \mathrm{D}$ finite element method on the objective model has been developed for the IRT on NDT, to confirm the possibility of IRT fitful work performance of NDT, compared the conventional

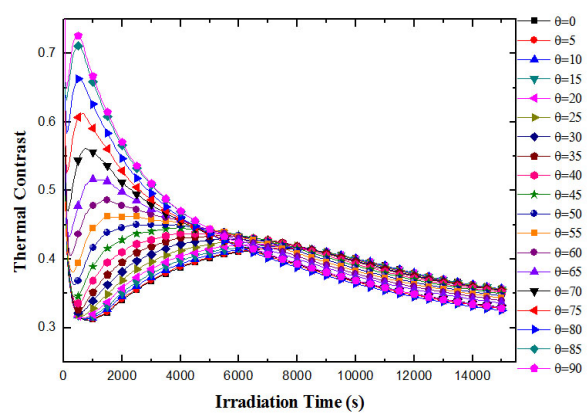

Fig. 9 The thermal contrast evolution in time for different angled defects

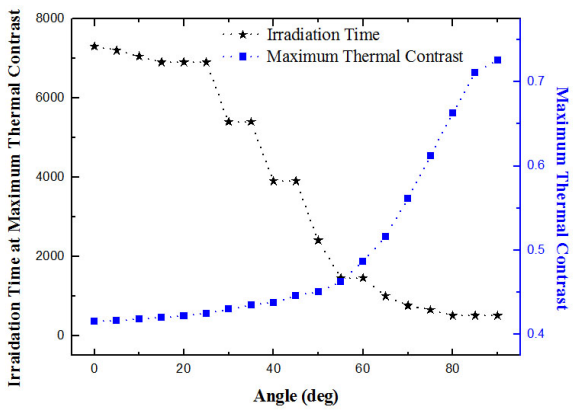

Fig. 10 The maximum thermal contrast and its corresponding irradiation time variations with defect orientations

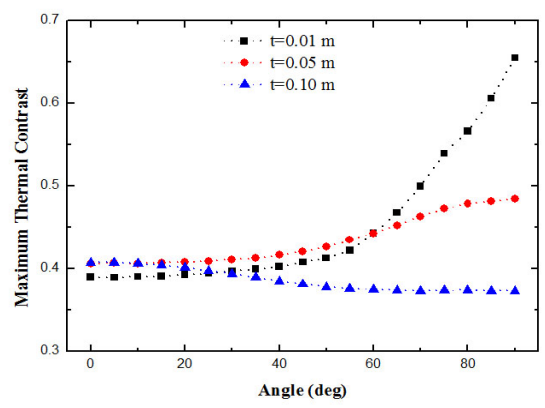

Fig. 11 The maximum thermal contrast variation with different orientations of different thickness

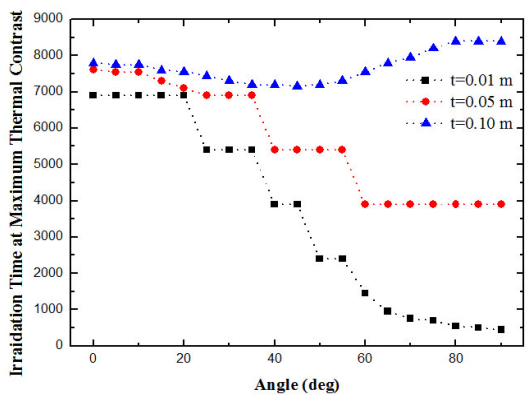

Fig. 12 The irradiation time of maximum thermal contrast variation of different thickness 
method of NDT which has the obvious drawbacks. A model contains a plate with an internal defect and two halogen lamps. The temperature profile based on the different of irradiation time is investigated and there is a maximum thermal contrast at a certain time, which is very important to have an effective inspection in the IRT process. The numerical simulation results show that IRT is helpful to optimize the experimental conditions and show the competitive potential, compared with the conventional NDT methods.

Parametric studies on the defect geometry have been implemented. The maximum thermal contrast is reversed with the defect depths, while its corresponding irradiation time shows a linear relation. The defect thickness shows a small difference on the thermal contrast involution in time domain. The thermal contrast increases with the increased defect thickness and then become saturated at some level. Different orientations of thin defects can be inspected with IRT, which shows high thermal contrast. IRT could make the detection on the thick defect inspection, but it is its weak point.

The numerical simulation result is helpful to optimize the experimental conditions and explain the experimental data of IRT.

\section{Acknowledgement}

This work was supported by the National Research Foundation of Korea (NRF) grant, which is funded by the Korea government (MEST) (No. 2011-0020024).

\section{References}

[1] D. J. Titman, "Applications of thermography in nondestructive testing of structures," NDT \& E International, Vol. 34, No. 2, pp. 149-154 (2001)

[2] C. I.-Castanedo, M. Genest, J.-M. Piau,
S. Guilbert, A. Bendada and X. P. V. Maldague, "Active infrared thermography techniques for the non-destructive testing of materials," Chapter XIV of the book "Ultrasonic and advanced methods for nondestructive testing and material characterization," Ed. Chen CH, pp. 325-348 (2007)

[3] H. S. Park and M. Y. Choi, "Research trends in infrared thermography NDT ultrasound infrared thermography technology," Journal of the Korean Society for Nondestructive Testing, Vol. 32, No. 3, pp. 307313 (2012)

[4] H. Park, M. Choi, J. Park, J. Lee, W. Kim and B. Lee, "Infrared thermography characterization of defects in seamless pipes using an infrared reflector," Journal of the Korean Society for Nondestructive Testing, Vol. 32, No. 3, pp. 284-290 (2012)

[5] L. Balaji, K. Balasubramaniam and C. V. Krishnamurthy, "Induction thermography for non-destructive evaluation of adhesive bonds," AIP Conference Proceedings, Vol. 1511, pp. 579 (2013)

[6] S. Shepard, Y. Hou, J. Lhota, D. Wang and T. Ahmed, "Thermographic measurement of thermal barrier coating thickness," Thermosense XXVII, Vol. 5782, pp. 167178 (2005)

[7] B. Cannas, S. Carcangiu, G. Concu and N. Trulli, "Modeling of active infrared thermography for defect detection in concrete structures," Proceedings of the 2012 COMSOL Conference (2012)

[8] C. C. Cheng, T. M. Cheng and C. H. Chiang, "Defect detection of concrete structures using both infrared thermography and elastic waves," Automation in Construction, Vol. 18, No. 1, pp. 87-92 (2008)

[9] T. Sakagami and S. Kubo, "Development of a new nondestructive testing technique for quantitative evaluations of delamination 
defects in concrete structures based on phase delay measurement using lock-in thermography," Infrared Physics and Technology, Vol. 43, pp. 311-316 (2002)

[10] N. Kim and Z. Lim, "Thermographic detection of surface crack using holomorphic function of thermal field," Journal of the Korean Society for Nondestructive Testing, Vol. 32, No. 3, pp. 296-301 (2012)

[11] Y. Cho, "A study on the optimized test condition of lock-in IR thermography by image processing," Journal of the Korean Society for Nondestructive Testing, Vol. 32, No. 3, pp. 276-283 (2012)

[12] M. Susa, C. Ibarra-Castanedo, X. Maldague and A. Bendada, "Pulse thermography applied on a complex structure sample: comparison and analysis of numerical and experimental results," IV Pan American Conference in END (2007)

[13] U. Sreedhar, C. V. Krishnamurthy, K. Balasubramaniam, V. D. Raghupathy and S. Ravishankar, Modeling and Simulation for Temperature Prediction in Welding Using Infrared Thermography, Proceedings of the National Seminar \& Exhibitionon Non-Destructive Evaluation NDE, pp. 10-12 (2009)

[14] X. P. Maldague, "Theory and Practice of Infrared Technology for Nondestructive Testing," John Wiley \& Sons, Inc., New York (2001) 